\title{
SILICON BULK MICROMACHINED VIBRATORY GYROSCOPE
}

\author{
Tony K. Tang, Roman C. Gutierrez, Jaroslava Z. Wilcox, Christopher Stell, \\ Vatche Vorperian, Robert Calvet, Wen J. Li, Indrani Charkaborty, Randall Bartman \\ Jet Propulsion Laboratory \\ MicroDevices Laboratory \\ California Institute of Technology \\ 4800 Oak Grove, Pasasena, CA $91109-8099$ \\ William J. Kaiser \\ Department of Electrical Engineering \\ University of California, LA, CA 90024-1594
}

\begin{abstract}
This paper reports on design, modeling, fabrication, and characterization of a novel silicon bulk micromachined vibratory rate gyroscope designed for microspacecraft applications. This new microgyroscope consists of a silicon four leaf clover structure with a post attached to the center. The whole structure is suspended by four thin silicon cantilevers. This device is electrostatically actuated and detects Coriolis induced motions of the leaves capacitively. A prototype of this microgyroscope has a rotation responstivity (scale factor) of $10.4 \mathrm{mV} / \mathrm{deg} / \mathrm{sec}$ with scale factor nonlinearity of $<1 \%$, and a minimum detectable noise equivalent rotation rate of $90 \mathrm{deg} / \mathrm{hr}$. at an integration time of 1 second. The bias stability of this microgyroscope is better than $29 \mathrm{deg} / \mathrm{hr}$. The performance of this microgyroscope is limited by the electronic circuit noise and drift. Planned improvements in the fabrication and assembly of the microgyroscope will allow the use of Q-factor amplification to increase the sensitivity of the device by at least two to three orders of magnitude. This new vibratory microgyroscope may have applications in inertial navigation and attitude control. It offers potential advantages of almost unlimited operational life, high performance, extremely compact size, low power operation, and low cost.
\end{abstract}

\section{INTRODUCTION}

Future spacecraft and rovers will require high performance microgyroscopes that are compact and cost effective with low power consumption for attitude and maneuver control, tumble recovery of spacecraft, and stabilization and pointing of instruments such as cameras, antenna, detectors, and solar panels. Microgyroscopes with 0.1 to $10 \mathrm{deg} / \mathrm{hr}$ bias stability performance can also be used to supplement other external inertial reference systems for spacecraft such as the Global Positioning System (GPS), sun sensor, or star tracker by "filling in the gaps" during times the direct use of these systems is not possible [1]. For example, a sun sensor cannot be used during an eclipse, or during periods of several minutes between star sightings by a narrow field-of-view star tracker, or when a GPS navigation signal suffers short term dropouts due to inadequate antenna coverage or physical or electromagnetic interferences. These new microgyroscopes not only will be relevant for various extraterrestrial missions, such as planetary and interplanetary astrophysics research but will also have terrestrial applications in automotive navigation systems, autonomous control of underwater and land vehicles, platform stabilization, and smart guidance for munitions, missiles, and robotics.

This paper reports on the development of a novel silicon bulk micromachined vibratory gyroscope that is under development for microspacecraft attitude and navigation control applications. This new microgyroscope offers several features, including: 1) the use of simple bulk silicon technology which provides precise structures, increases yield, and reduces cost;2) high rotation rate sensitivity due to the low frequency resonator design, differential mode of operation, and lock-in amplification detection; 3) low loss resonator to provide high quality (Q) factor for mechanical amplification. and 4) symmetric resonator design with degenerate modes. The design, fabrication, modeling, experimental characterization, and packaging of the microgyroscope for spaceflight experiment will be described in the next sections, followed by a conclusion.

\section{DESIGN}

In a vibratory gyroscope, the rotation sensing mechanical element is initially driven to oscillate in one mode (called input or drive mode $[\mathbf{2 , 3}, \mathbf{4}]$. Under rotation, Coriolis acceleration induces energy transfer from the input mode to another oscillatory mode (output or sense mode) causing the sensing element to precess. The best performance for the vibratory gyroscope is obtained when the two oscillatory modes (drive and sense) have the same resonant frequency with high quality (Q) factor. The response to the Coriolis acceleration is then amplified by the $\mathrm{Q}$ factor of the resonance resulting in improved sensor performance.

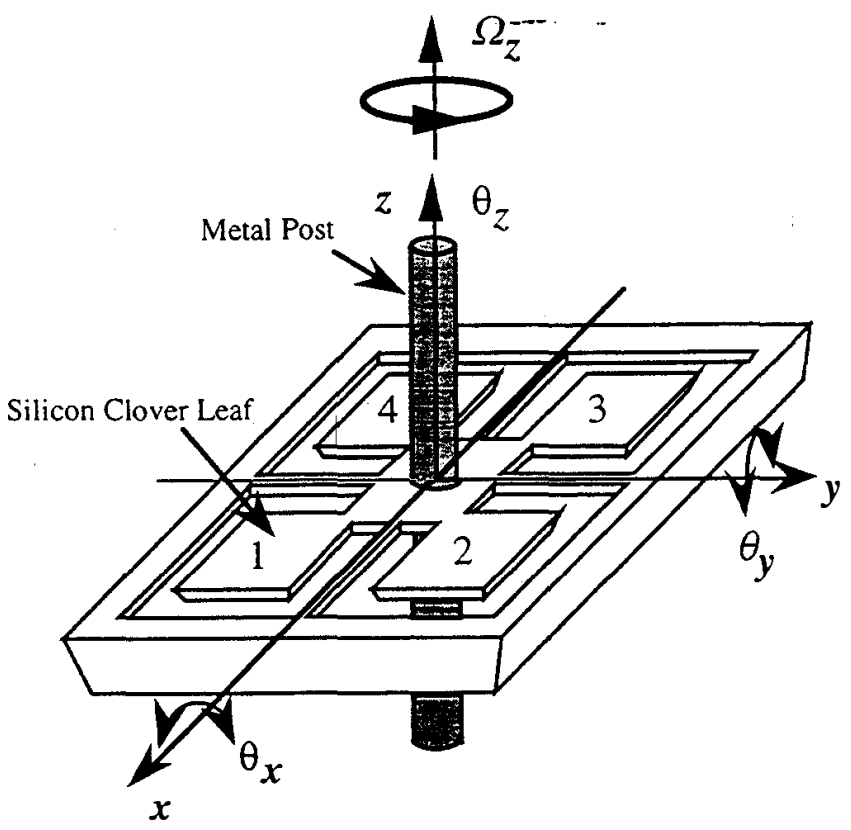

Fig. 1 Schematic of the clover-leaf microgyroscope. 
The JPLUCLA microgyroscope resembles a four leaf clover suspended by four thin wires from the gyroscope housing. The rim, the clover structure and the suspensions are made from single crystal silicon (Fig. 1). A metal post is rigidly attached through the center of the structure in the direction perpendicular to the plane of the clover leaves. The four suspensions provide mechanical support and restoring force for the harmonic motion of the whole structure. The clover leaf structure and the metal post have a combined mass of about $10^{-5}$ $\mathrm{kg}$. The mechanical resonant frequencies of the microgyroscope vary according to design in the 300 to $500 \mathrm{~Hz}$ range. The low resonant frequency is due to the large mass of the post and soft suspensions. Since the response to the gyroscope is inversely proportional to the resonant frequency, low resonant frequency increases the device responsitivity.

The silicon clover-leaf structure with the post is bonded to a quartz base plate patterned with gold electrodes. The clover leaves provide large areas for electrostatic driving and capacitive sensing. The resonator is excited by applying an AC voltage to the capacitors associated with leaves 2 and 3 , and this excites the rotation $\theta_{x}$ about the $x$ axis. Since the resonator is symmetrical, $\theta_{x}$ and $\theta_{y}$ are degenerate with rocking-like displacement for the clover leaves. The degeneracy of the drive and sense modes allows Q-factor amplification of modal displacements. Q-factor amplification of the drive and sense motion reduces drive voltage and increases the sensitivity of the device respectively. The Q-factor of this symmetric resonant structure can be very high since it can be perfectly balanced with a fixed center of gravity, and the sum of forces and moments resulting from the vibration modes can be zero.

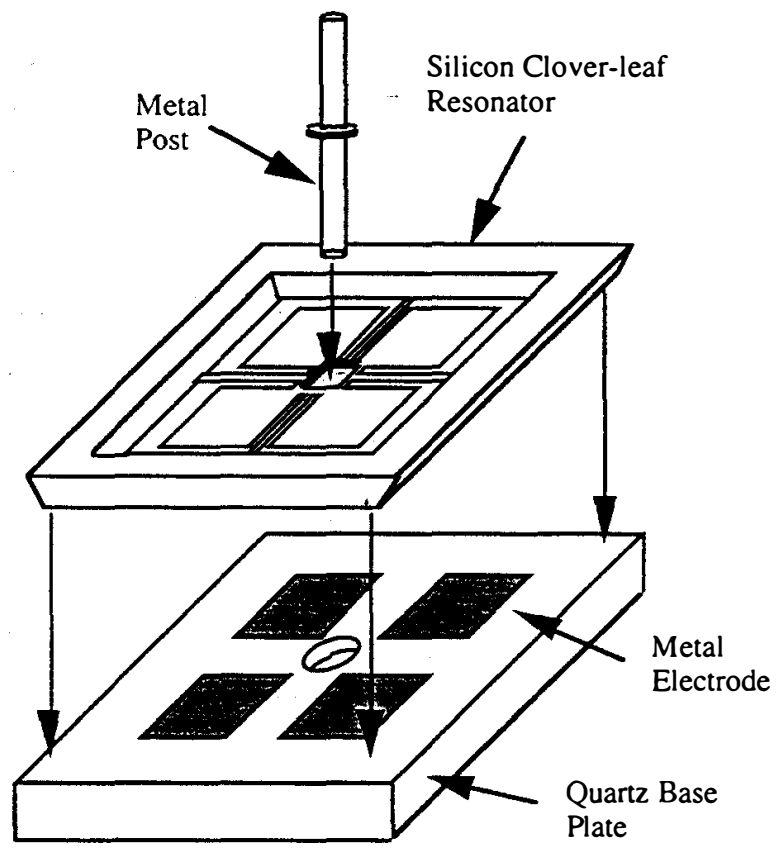

Fig. 2 Exploded view of the clover-leaf microgyroscope.

Since the post is rigidly attached to the clover-leaf structure, the movements of the clover leaves translate to movements of the post. When the leaves oscillate in the drive mode, the displacement of the post is near parallel to the clover leaf surface in the $y$-direction. When a rotation rate is applied about the z-axis. Coriolis force acts on the oscillating post causing it to displace in the orthogonal direction (i.e. $x$ - direction). This post displacement is translated back into the rocking motion $\theta_{y}$ of the clover leaves. The post provides a large Coriolis coupling which transfers energy between the two orthogonal rocking modes.

\section{FABRICATION}

The structure of the silicon microgyroscope is shown in Fig.2. The microgyroscope consists of a quartz base plate patterned with metal electrodes, and the silicon clover-leaf resonator suspended from the thick silicon rim. The quartz base plate and the silicon rim are bonded together by using low shrinkage UV curable epoxy. The dimension of the silicon clover-leaf resonator is $7 \mathrm{~mm} \times 7 \mathrm{~mm}$, the dimension of each clover leaf is $1.1 \mathrm{~mm} \times 1.1 \mathrm{~mm}$, and the post is 500 microns in diameter and $5 \mathrm{~mm}$ in length. The quartz base plate is a square 1 $\mathrm{cm} \times 1 \mathrm{~cm}$ and approximately 400 microns thick. The sensing capacitors of the microgyroscope are formed between $\mathrm{Cr} / \mathrm{Au}$ electrodes on the quartz base plate and the silicon clover leaves. The gap between the base electrodes and the clover leaves is about 10 to 20 microns.

The fabrication of the silicon clover-leaf resonator requires only two masking and etching steps using bulk micromachining technology. The fabrication process sequence for the microgyroscope is shown in Fig.3a. Fabrication begins with the standard n-type $<100\rangle$ double side polished silicon wafer that has two silicon epilayers grown on top. The bottom layer is a 4 micron $p+\left(10^{20}\right.$ boron doped) etch stop layer. The top layer is a 26 micron n-type silicon epilayer. A $1000 \AA$ layer of thermal silicon dioxide is grown on the wafer and patterned with the clover leaf pattern. This patterned oxide layer is used as a mask for EDP etching of the 26 micron silicon epilayer to the $\mathrm{p}+$ etch-stop layer. All features of the clover-leaf pattern run along the crystal planes, resulting in very smooth shapes. This is due to the selectivity of the anisotropic EDP etch which automatically provides very precise dimensions and shape for the suspensions. This is very important for the microgyroscope performance since the mode shape and resonant frequencies are affected by any device asymmetries.

An SEM picture of the etched profiles of the clover leaf structure is shown in Fig. 4. After the wet etch the thermal oxide mask is stripped away in buffered HF. This is followed by a $3000 \AA$ thick thermal silicon dioxide. The silicon dioxide on back of the microgyroscope is then patterned and the device is again etched in EDP. This etch removes almost all the silicon substrate and stops at the p+ etch-stop layer. Next, the wafer is cleaved into individual devices and separated. The cleaved devices are then etched in 1:3:8 $\mathrm{HNA}\left(\mathrm{H}_{2} \mathrm{O}_{2}: \mathrm{HNO}_{3}: \mathrm{CH}_{3} \mathrm{COOH}\right)$ until the $\mathrm{p}+$ stop-etch layer is removed. Finally, the oxide layer is striped away in buffered HF. The removal of both the $p+$ layer and the silicon dioxide layers removes stress gradient which can cause warpage of the clover leaf structure.

The fabrication process of the quartz base plate consists of two lithography and etching steps as shown in Fig.3b. A hole is first drilled into the quartz base plates for the post. This is followed by the patterning and etching of the quartz base to the depth of 10 to 20 microns using HF. This provides the gap spacing that separates the silicon clover leaves and the electrodes on the quartz plate. Next, a $\mathrm{Cr} / \mathrm{Au}$ metallization is evaporated onto the whole quartz plate, and is etched to give the electrodes patterns. Then, the quartz plate is diced into individual devices. The silicon resonator and the quartz base plate are then bonded together using UV curable epoxy. All devices are first tested for resonant frequencies and mode shapes. 
After these initial tests, a metal post is epoxied into the hole on the silicon resonator (Fig. 5).

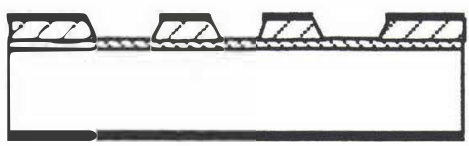

1) 1st thermal oxide growth and patterning, topside silicon epilayer EDP etch.

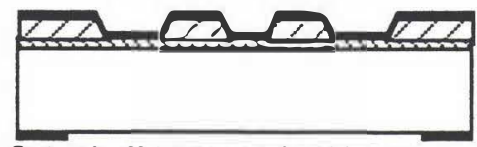

2) Striped off 1 st thermal oxide, 2nd oxide growth and patterning.

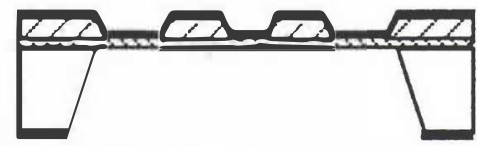

3) Backside EDP etch.

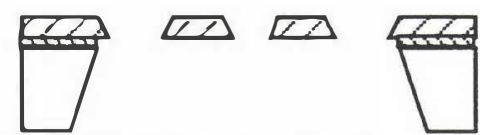

4) Backside HNA etch and oxide etch.
$\mathrm{SiO}_{2}$
$\mathrm{Si}$
จ $p+$ silicon epilayer
E. $n$ silicon epilayer

Fig. 3a Fabrication process of the silicon clover-leaf structure.

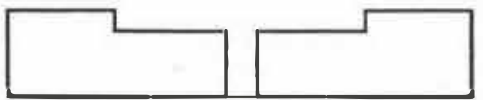

1) HF etching of the quartz base plate.

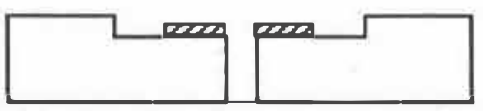

2) Metallization of the quartz base plate.

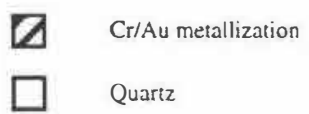

Fig. 3b Fabrication process of the quartz base plate.

\section{CONTROL AND READOUT ELECTRONICS}

In order to design the electronics for actuating and sensing the microgyroscope, an electrical model of the microgyroscope is derived and analyzed. The model described in this section gives a complete description of the frequency response of the microgyroscope. Important parasitic effects, such as interplate capacitances and mechanical coupling between the orthogonal modes under no rotation, are included in the model. Fig. 6 shows the basic electrical model which gives the excitation-to-sense transfer function, $\hat{v}_{2} / \hat{v}_{1}$ or $\hat{v}_{3} / \hat{v}_{4}$, of the microgyroscope taking one pair of opposite plates at a time. The mechanical resonance is represented by the series $L_{\mu} C_{\mu} R_{\mu}$ resonant circuit which is excited via the dependent sources $\delta \hat{v}_{1}$ and $\delta \hat{v}_{2}$ which communicates with the port voltage $\hat{v}_{1}$ and $\hat{v}_{2}$ respectively ( $\delta$ represents the deflection/volt in the plates at zero frequency).

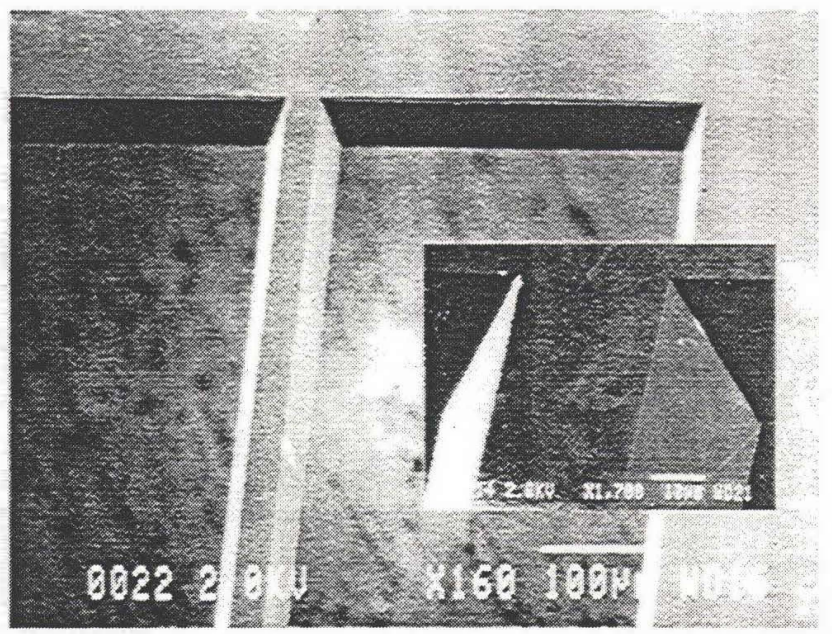

Fig. 4 SEM photograph of the corner between the suspension and the center of the clover-leaf structure demonstrating the sharpness and precision of the etching process.

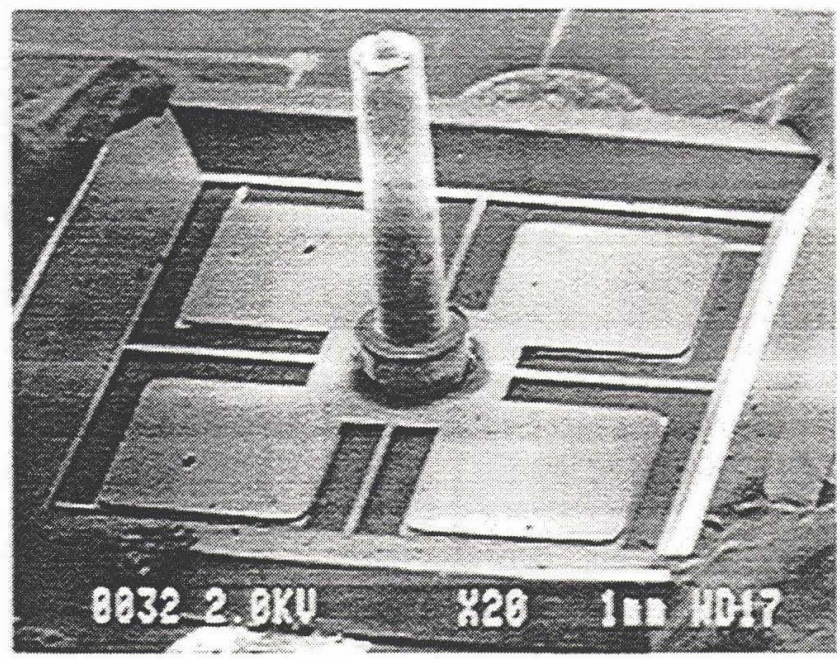

Fig. 5 SEM photograph of an assembled microgyroscope

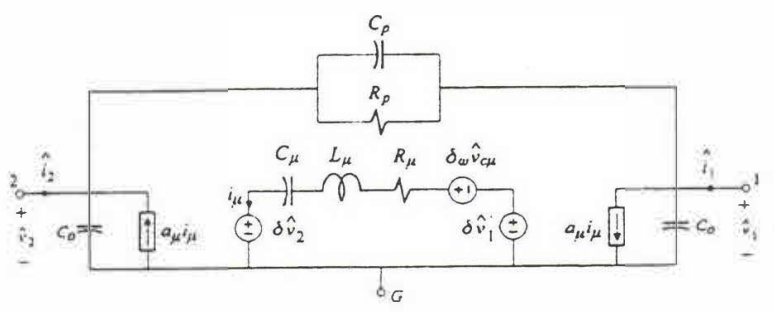

Fig. 6 Equivalent circuit model for an opposite pair of plates.

The effect of the mechanical motion on the electrical ports in turn is given by the dependent sources $a_{\mu} i_{\mu}$. The interplate 
parasitic resistance and capacitance are given by $R_{p}$ and $C_{p}$ respectively. The dependent source $\delta_{\omega} \hat{v}_{c \mu}$ is the Coriolis induced term which is further explained in the next paragraph. The transfer function predicted by this model (with $\omega=0$ and, hence, $\delta_{\omega}=0$ ) is shown in Fig.7 in which the notch at $\omega_{n}$ is mainly due to the parasitic capacitance $C_{p}$.

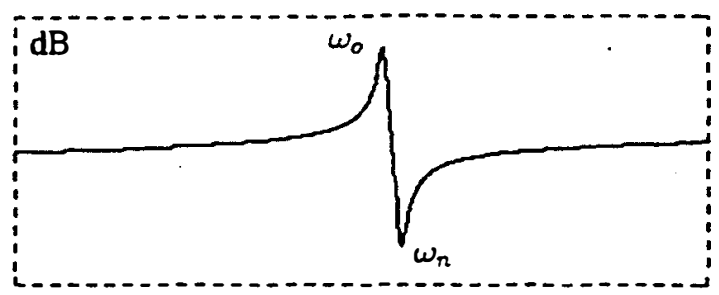

Fig. 7 Excitation-to-output transfer function obtained from the model in Fig. 6.

Fig. 8 shows the experimental transfer functions of each pair of opposite plates of a microgyroscope whose orthogonal resonant modes (drive and sense) are at $445 \mathrm{~Hz}$ and $435 \mathrm{~Hz}$ respectively. The departure of each of these transfer functions from the one shown in Fig. 7 for a single pair of plates is primarily caused by parasitic electrical and mechanical coupling between the orthogonal modes when the gyro is at rest and by the slight difference between the $\delta$ and $a_{\mu}$ for each pair of plates. These curves can now be easily predicted when a full model of the gyro is constructed by assembling together the model for each pair of plates given in Fig. 6 as shown in Fig. 9 . The parasitic mechanical coupling between the drive and sense modes when $\omega=0$ is modeled by $\delta_{d s} \hat{v}_{\mu d}$ and $\delta_{s d} \hat{v}_{j \boldsymbol{\mu} s}$ while the Coriolis term is given by $\delta_{\omega} \hat{v}_{\mu d}$. The predictions of this model (with $\omega=\delta_{\omega}=0$ ) are shown in Fig. 10 and are seen to match well with those in Fig. 8. Detailed analytical expressions derived from the circuit in Fig. 9 will be given in a future paper.

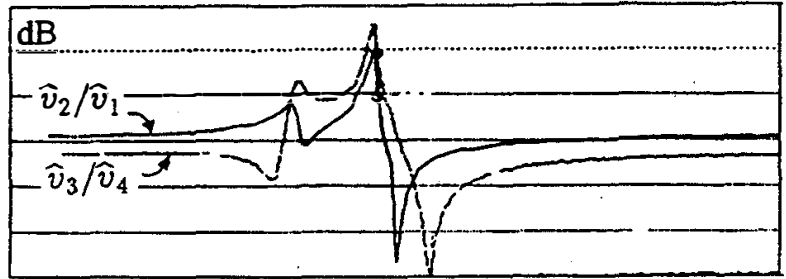

$400 \mathrm{~Hz}$

$500 \mathrm{~Hz}$

Fig. 8 Experimental plots for the excitation-to-output transfer function.

The actuation, or drive, circuit is realized by designing an oscillator $(H(s))$ around the microgyroscope which locks in onto the drive resonance mode as shown in Fig. 11. The purpose of summing the signals from both sense plates is to remove the differential signal between them and hence the response of the sense resonance from the feedback loop. The sense circuit, on the other hand, subtracts the signals from both sense plates in order to remove the common-mode drive signal. A lock-in amplifier is used in order to detect a differential signal induced due to rotation.

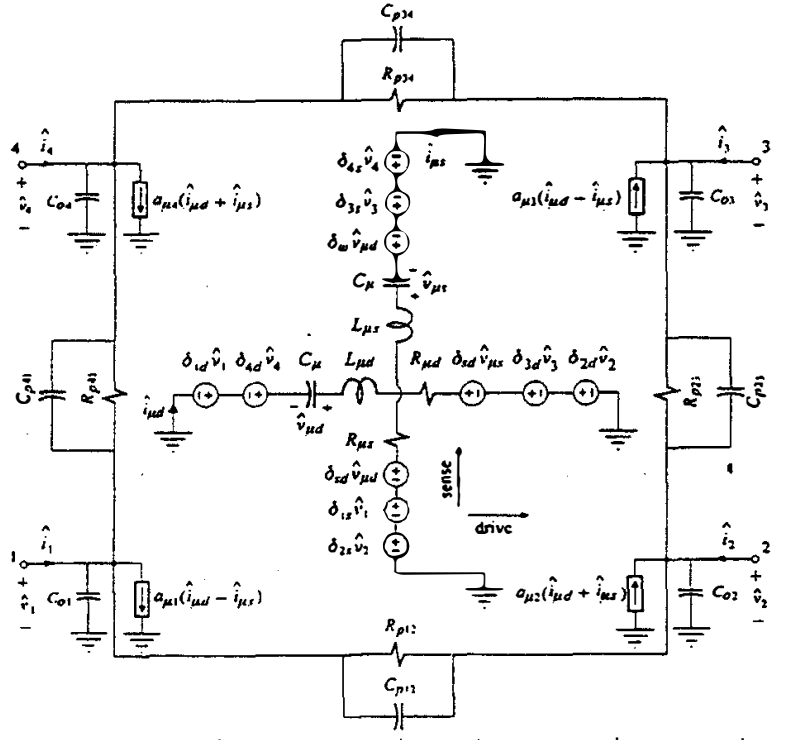

Fig. 9 Complete electrical model of the microgyroscope.

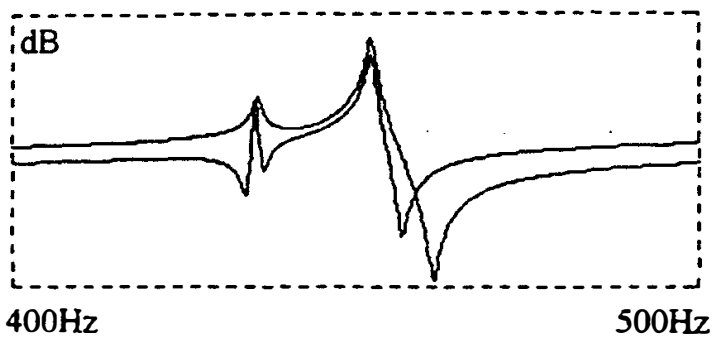

Fig. 10 Transfer function obtained from model in Fig. 9.

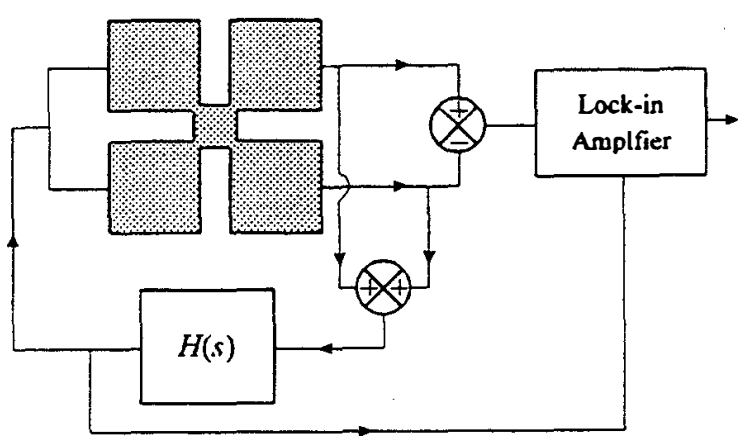

Fig. 11 Exciting and sensing of the microgyroscope.

\section{MODELING}

The Lagrange equations of motion are written for the angular displacements $\theta_{x}, \theta_{y}$, and $\theta_{z}$ measured in the coordinate system that rotates with the microgyroscope housing. The center $O$ is assumed stationary, $\theta_{z}$ is rotation around the post ( $z$ ) axis, and $x$ and $y$ are the spring axes. Thus, in this system, $\theta_{x}$ and $\theta_{y}$ represent measurable, near-vertical angular displacements. For spin about the $z$ axis, $\theta_{z}$ remains decoupled from $\theta_{x}$ and $\theta_{y}$, and for small $\theta_{x}, \theta_{y}$, and $\Omega_{z} \omega$, the cloverleaf 
displacements for a quad-symmetric structure driven by the application of the torque $\mathrm{T}$ to the leaves 2 and 3 , are described by the following approximate equations of motion:

$$
\begin{aligned}
& 2 I_{x x} \ddot{\theta}_{x}-4 I_{x} \Omega_{z} \dot{\theta}_{y}+\kappa_{x} \theta_{x}+\alpha_{x} \dot{\theta}_{x} \approx T(t) \\
& 2 I_{y y} \ddot{\theta}_{y}-4 I_{z} \Omega_{z} \dot{\theta}_{x}+\kappa_{y} \theta_{y}+\alpha_{y} \dot{\theta}_{y} \approx 0
\end{aligned}
$$

where the terms proportional to $\alpha$ 's and time derivatives $\dot{\theta}_{x}$ and $\dot{\theta}_{y}$ describe vibration damping, and $\mathrm{T}(t)$ is the driving torque. The "mass moment" is $l_{z} \equiv \sum_{i} z_{i}^{2}\left(m_{i} / 2\right)$ where $z_{i}$ is the $z$ component of $\vec{r}_{i}$ from $O$ to any mass element of the moving structure. The other moments of inertia are :

$$
\begin{aligned}
& I_{x x} \equiv I_{z}+I_{y}=\sum_{i}\left(z_{i}^{2}+y_{i}^{2}\right)\left(m_{i} / 2\right) \\
& I_{y y} \equiv I_{z}+I_{x}=\sum_{i}\left(z_{i}^{2}+x_{i}^{2}\right)\left(m_{i} / 2\right) .
\end{aligned}
$$

For the structure with the post, M5 $\gg(M 1, M 2, M 3, M 4)$, and $I_{x x} \cong I_{y y} \cong I_{z} \cong\left(M_{5} / 2\right) L_{5}^{2}$, where $L_{5}$ is the arm length from $O$ to the mass center of $M_{5} / 2$ and $M_{1-4}$ are the masses of the clover leaves and $M 5$ is the mass of the post. The restoring torque parameters $\kappa_{x}$ and $\kappa_{y}$ relate to "spring" constants for linear displacements due to tension and twist, and to the device dimensions.

The homogeneous solutions to Eq. 1 and 2 are the normal modes. For small damping in the absence of platform rotation, the modes oscillate with the resonant frequencies $\omega_{x} \cong \sqrt{\kappa_{x} / 2 I_{x x} \text { and } \omega_{y} \cong \sqrt{\kappa_{y} / 2 I_{y y}}}$ for $\theta_{x}$ and $\theta_{y}$ rotations, respectively. The application of AC voltage to the capacitors associated with leaves 2 and 3 (Fig. 1) drives the vibrational rotation about the $x$-axis (angular displacement $\theta_{x}$ ). Upon application of input rotation $\Omega_{z}$, the vibrational energy is transferred from the $\theta_{x}$ to the $\theta_{y}$ vibrational mode. We consider $\mathrm{T}(t)=\mathrm{T}_{o} \cos (\omega t)$ that drives $\theta_{x}$, at $\omega \gg>\Omega_{z}$. Since $\theta_{x}>>\theta_{y}$, by neglecting the Coriolis term in Eq. 1, the driven $\theta_{x}$ is:

$$
\theta_{x} \approx \theta_{x o} \sin \left(\omega t+\psi_{x}\right)
$$

where the amplitude

$$
\theta_{x o}=\frac{T_{o}}{2 I_{x x}} \frac{1}{\sqrt{\left(\omega^{2}-\omega_{x}^{2}\right)^{2}+\frac{\omega^{2} \omega_{x}^{2}}{Q_{x}^{2}}}} \rightarrow \frac{T_{o}}{2 I_{z}} \frac{Q_{x}}{\omega_{x}^{2}} \rightarrow \frac{T_{o}}{\kappa_{x}} Q_{x}
$$

where the last two expressions on the right hand side apply for $\omega$ near $\omega_{x}$, i.e., $\left|\omega-\omega_{x}\right|<\gamma_{x}, \gamma_{x} \cong \alpha_{x} / 2 I_{x x} \cong \omega_{x} / Q_{x}$. At times $\gg>l / \gamma_{x}$, after the application of $\Omega_{z}$, and the transient effects have leveled-off, the spin-induced $\theta_{y}$ is given by the inhomogeneous solution of Eq.2:

$$
\theta_{y}=\theta_{y_{0}} \cos \left(\omega t+\psi_{y}\right)
$$

where

$$
\theta_{y o}=\theta_{x o} \frac{I_{z}}{I_{y y}} \frac{2 \Omega_{z}}{\omega_{y}} \frac{\omega \omega_{y}}{\sqrt{\left(\omega^{2}-\omega_{y}^{2}\right)^{2}+\frac{\omega^{2} \omega_{y}^{2}}{Q_{y}^{2}}}} \rightarrow
$$

$$
\rightarrow \frac{4 I_{y y} \Omega_{z}{ }^{\omega \theta} x_{0}}{\kappa_{y}} Q_{y} \rightarrow \theta_{x o} \frac{2 \Omega_{z}}{\omega_{y}} Q_{y}
$$

where the last two expressions on the right hand side are valid for a heavy post ( $I_{y y} \cong I_{z}$, and $\omega$ near $\omega_{y}$ )and $Q_{y}$ is the $Q$ factor for the $\theta_{y}$ resonance. These results show that the degeneracy of the resonant frequency can enhance the spininduced $\theta_{y}$ by the product of both the driven and sensing $Q$ factors. This degeneracy can be accomplished with a symmetric configuration of the cloverleaf and springs resonator design.

\section{MEASUREMENT}

In order to fully analyze a micromachined vibratory gyroscope, it is necessary to carry out a significant amount of testing. In this case, two separate test stations were assembled; the first test station was designed to be used to quantify the mechanical properties of the gyroscope, and the second to measure the performance of our device as a rotation sensor.

The first test station consists of a small vacuum chamber with electrical probes to electrically excite the microgyroscope, and a Polytech OFV 501 laser vibrometer (Doppler velocimeter) to accurately measure mechanical motion. Some of the mechanical properties of the gyroscopes measured with this setup are: resonance frequencies, Q-factors, mode shapes, hysteresis, snap-down voltage, symmetry (by exciting different electrodes), operational voltage, and survivability (by driving it with a very large amplitude signal).

The second test station uses a Triotech S347B rate table with a resolution of 0.01 degrees per second to rotate a microgyroscope inside a vacium chamber. The motion of the microgyroscope is measured both electrically and with a Polytech OFV 501 laser vibrometer. Some of the performance characteristics measured using this apparatus are: the drive and up/down mode frequencies, the sense mode residual mechanical excitation (zero for a perfectly balanced gyro), the rotation responsitivity $(\mathrm{mV} / \mathrm{deg} / \mathrm{sec})$, vibrational amplitude, linearity of the response, the noise characteristics of the gyroscope (Green chart, PSD, autocorrelation), bias offset (output of the lock-in without rotation), bias drift, response to rotation when sense axis is perpendicular to rotation, and response to rotation when excited in the up/down mode.

Several finished microgyroscopes were tested inside the test stations. The resonant frequency of the drive mode is at $452 \mathrm{~Hz}$, and the sense mode is at $447 \mathrm{~Hz}$ for one of the devices. The Q-factor of the drive and sense mode is approximately 500 . Both resonant modes are rocking modes with nodal lines along the spring directions. The separation between the resonant frequencies is primarily due to stress from the bonding of the silicon clover leaf structure to the quartz structure and to fabrication errors. The microgyroscope was driven at the drive resonance for rotation testing. The response of this device does not have significant Q-factor amplification of the sense mode displacement due to splitting of the drive and sense resonant frequency. The driving voltage of the microgyroscope is approximately $4 \mathrm{~V}$ peak to peak. The snap-down voltage is approximately $20 \mathrm{~V}$. A plot of the output voltage vs. rotation rate is shown in Fig.12. From this figure, the measured responsitivity (scale factor) of the gyroscope is $10.4 \mathrm{mV} /(\mathrm{deg} / \mathrm{sec})$ with a scale factor nonlinearity of $<1 \%$ at an integration time of 1 second. 


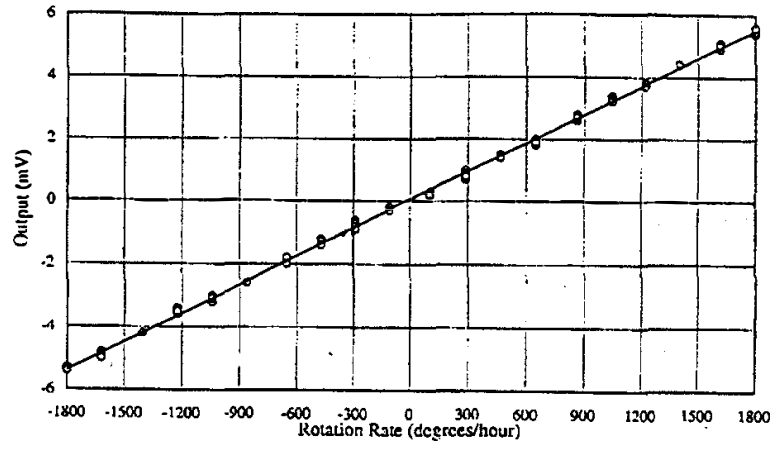

Fig. 12 Rotation rate responstivity of the clover-leaf microgyroscope.

The Allan deviation chart (Fig. 13) shows the lowest detectable noise equivalent rotation rate is about $90 \mathrm{deg} / \mathrm{hr}$ at an integration time of 1 seconds. The rise in the noise after the 15 second integration time is mostly due to drift in the electronics. Thus, the bias stability of this microgyroscope should be below $29 \mathrm{deg} / \mathrm{hr}$. The performance of this micro-gyroscope is limited by the electronic circuit. Closed-loop circuitry is being developed to improve the sensitivity of this microgyroscope by lowering the noise and reducing the drift. Improvements in the fabrication and assembly of the microgyroscope will allow the use of Q-factor amplification to increase the sensitivity of the device by at least two to three orders of magnitude. This will allow the clover-leaf microgyroscope to be useful in attitude and navigation control applications.

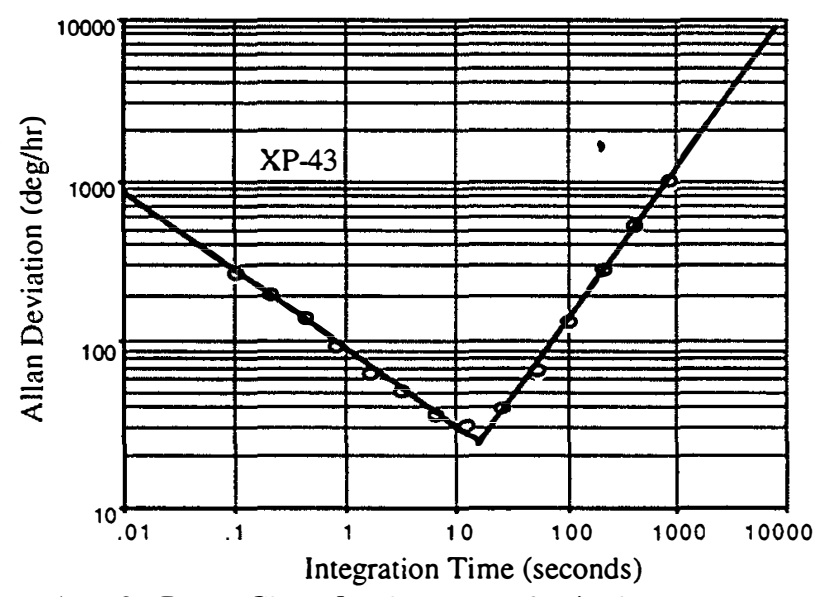

Fig. 13 Green Chart for the clover-leaf microgyroscope.

\section{CONCLUSION AND FUTURE PLAN}

This paper has reported on the design, modeling, fabrication, and characterization of a novel silicon bulk micromachined vibratory gyroscope designed for microspacecraft applications. A microgyroscope prototype that has been fabricated so far has a rotation responsitivity (scale factor) of $10.4 \mathrm{mV} / \mathrm{deg} / \mathrm{sec}$ with $<1 \%$ nonlinearity at an integration time of 1 second. The lowest detectable noise equivalent rotation rate is $90 \mathrm{deg} / \mathrm{hr}$ at an integration time of 1 second. The bias stability of this microgyroscope is at or better than 29 $\mathrm{deg} / \mathrm{hr}$. The performance of this microgyroscope is limited by the electronic circuit noise and drift, fabrication errors, and stress resulting from the assembly process. Improvements in these areas will further increase the sensitivity and improve the drift performance of this microgyroscope.
This JPLUCLA clover-leaf microgyroscope is planned as a piggyback spaceflight experiment called the Commercial Hardware Micro-Gyro $(\mathrm{CH} \mu \mathrm{G})$ on the Mars Pathfinder spacecraft to study re-entry dynamics. Each microgyroscope is individually packaged in $2.5 \times 3 \times 0.4$ inches stainless steel case. Four such packages are assembled onto the mother board which consists of a thermal plate and the digital electronics for communication with the spacecraft. The participation of the JPLUCLA clover-leaf microgyroscopes in the CHuG spaceflight experiment has provided valuable experiences in the integration, packaging, and space qualification of Micromachined ElectroMechanical System (MEMS) microdevices for spaceflight missions.

The immediate goals for the JPLUCLA microgyro effort in the next few years include 1) improve the microgyroscope design to achieve 1-10 deg/hr bias stability performance, 2) produce a three-axes Micro-Inertial Measurement Unit (MIMU) consisting of hermetically sealed microgyroscopes packaged with micro-accelerometers for spaceflight validations in spacecraft, 3) investigate the issues relevant to the space qualification of the microgyros, such as device lifetime, device packaging, and survivability during launch and in the space environment.

In conclusion, we presented a new vibratory microgyroscope that offers potential advantages of almost unlimited operational life, high performance, extremely compact size, low power operation, and low cost for applications in inertial navigation and attitude control of microspacecraft.

\section{ACKNOWLEDGMENTS}

The authors thank Dr. Linda Miller for encouragement and support. We give special thanks to Dr. Paul Maker and Richard Müller for the fabrication of e-beam photolithographic masks. The work described in this paper was performed by the Center for Space Microelectronics Technology, Jet Propulsion Laboratory, California Institute of Technology, and was sponsored by National Aeronautics and Space Administration, Office of Space Access and Techology.

\section{REFERENCE}

[1] G. E. Sevaston, L. Craymer, and W. Breckenridge, "GPS Based Attitude Determination for Spacecraft: System Engineering Design Study and Ground Testbed Results", 19th Annual AAS Guidance and Control Conference, February 7-11, 1996, Breckenridge, CO, AAS 96-16, pp. 1 - 20.

[2] M.W. Putty, and K. Najafi, "A Micromachined vibrating Ring Gyroscope", Technical Digest of the 1994 Solid-State Sensor and Actuator Workshop, Hilton Head Isl., SC, 6/1316/94, Transducer Research Foundation, Cleveland (1994), pp. $213-220$

[3] J. Bernstein, S. Cho, A.T. King, A. Kourepenis, P. Maciel, M. Weinberg," A Micromachined Comb-Drive Tuning Fork Gyroscope," Digest IEEE/ASME Micro ElectoMechanical Systems (MEMS) Workshop, Ft. Lauderdale, Fl., pp. 143-148, February 1993.

[4] P. B. Ljung, T. N. Juneau, and A. P. Pisano, "Micromachined Two Input Axis Angular Rate Sensor", Proceedings of the ASME Dynamic Systems and Control Division, DSC-Vol. 57-2, 1995 IMECE/ASME, pp. 957-962. 